\title{
Research on Advertising Communication of Vertical Screen Short Video under Mobile Media
}

\author{
Le Yuan* \\ Tingyi Sunac Culture Communication Co., Ltd. E-mail: yuanle@sina.com
}

\begin{abstract}
In the era of mobile media, the application of smart phone is becoming more and more popular, and people spend a lot of time on mobile media every day. The advertisement dissemination based on smart phones, such as vertical short video advertisements, has always been extremely concerned by users and advertisers.
\end{abstract}

Keywords: Mobile Media; Vertical Screen Short Video Advertisement; Communication Studies

\section{Introduction}

With the popularity of smart phones and the arrival of mobile media era, traffic and network speed have become factors that affect people's choice of resources and information, changing the usage habits of mobile devices of target audiences and watching more video content on mobile devices. According to InMobi's "China Mobile Video Advertising Research Report 2019”, in 2017 and 2018, the spending of brand advertisers on mobile video advertising increased by 348\%. The mobile video advertising rate of all kinds of advertisements is increasing every year, and the proportion of video advertising expenditure in China has doubled. It is not difficult to see from the report that the growth of video advertising expenditure is mainly due to the growth of mobile video advertising expenditure, and vertical short video advertising is the main driving force for the growth of mobile video advertising. At present, the internal short video social platform, such as Vibrato, has quickly become the main position of short video advertising in mobile phone vertical screen advertising, leading the trend of short video advertising. It has also studied and completed the first local Creative Manual of Vertical Screen Advertising in Vibrato, and released the Case Manual of Douyin Short Video Marketing, which summarizes the experience of vertical screen short video advertising and provides ideas for many people who want to participate, laying the foundation for future development and exploration. Vertical-screen short video advertisements are constantly in the eyes of advertisers, and become a new form of video advertisements. In this paper, the vertical screen short video advertisement is a very short time in the mobile media era, which relies on mobile intelligent devices and short video platform to carry out the vertical screen version and spread the video advertisement information.

Advertising itself is an act of spreading information. To a certain extent, we can regard it as a communication activity to spread commodity information and content, thus influencing consumers' thoughts or behaviors. Media, audience and content of advertisement are important factors that affect the advertising effect, and they correspond to the media, audience and content in communication studies. Data show that the completion rate of mobile media playing is 9 times higher than that of vertical video advertisement, while the visual attention rate of horizontal video advertisement is 1.44 times higher than that of horizontal version, the interactive effect is 2 times higher than that of horizontal

Copyright (C) 2020 Le Yuan

doi: $10.18686 / \mathrm{mcs} . v 2 i 4.1361$

This is an open-access article distributed under the terms of the Creative Commons Attribution Non-Commercial License

(http://creativecommons.org/licenses/by-nc/4.0/), which permits unrestricted non-commercial use, distribution, and reproduction in any medium, provided the original work is properly cited. 
version, and the click rate is $41 \%$ higher than that of horizontal version. From the data and investigation, the vertical screen short video advertisement has obvious communication advantages compared with the general video advertisement, and this paper will analyze its communication advantages.

\section{The media of vertical screen short video advertising in the era of mobile media}

Mobile terminals, represented by smart phones, tablet computers and other devices, are the means of communication for vertical short video advertisements in the mobile media era. Mobile media has two following functions.

\subsection{Communication in the mobile scene}

Thanks to relevant information, in 2014, Chinese adults spent no more than 2 hours using mobile phones, tablets and other mobile terminals on the Internet, but after 2019, the Internet time almost doubled to about 4 hours, and the total time spent browsing mobile videos on the Internet created favorable conditions and the development of a large number of short video advertisements on mobile phones. The spread of mobile scenes is an important feature of today's mobile media, which helps people learn more through short videos in idle and fragmentary time. As long as people need it, it can be said that the vertical short video advertisement will lead to the satisfaction of people's needs. Compared with the traditional advertising communication mode, this mobile media-based advertising communication mode can bring people a good participation experience, arouse people's interest in tracking, and be willing to invest time and energy, while breaking the time and space constraints.

\subsection{Vertical screen}

Vertical screen also has the characteristics of mobile media, and has the functions belonging to all mobile media. Even in use, people can choose between vertical screen and horizontal screen according to their own needs, but most mobile media users don't like horizontal screen display, and more than $50 \%$ of users will lock the screen, thus avoiding the mobile phone from vertical screen to horizontal screen.

A study by an American research institute showed that $53 \%$ of the users in the survey sample did not use the horizontal screen to watch videos, which was roughly consistent with the daily observation results of the author. In real life, few people around us use the horizontal screen to watch videos, even if the vertical screen has black edges, they can't enjoy the full screen effect. This is because it is very troublesome to change the vertical screen to the horizontal screen. Through the above analysis, it can be found that under the influence of various habits; vertical screen display has been accepted and become a well-known video viewing mode. At present, short video social platforms at home and abroad are based on this particularity, and introduce vertical screen display full screen technology to meet people's viewing experience. With support of this technology, people can enjoy the visual effect of horizontal screen when watching on vertical screen. If there is special need, people can enlarge the details of video, which also promotes the spread of advertisements and can increase the visual effect of products on mobile phone screens.

\section{The audience}

\subsection{Huge audience}

At present, we mainly rely on various short video social platforms, such as Douyin, Kuaishou and Weishi, to display and use vertical short video advertisements in mobile media. These platforms communicate and socialize through short video content on vertical screens. Traffic scale is the number of media accessed and used, which is mainly affected by the number of users and the degree of environmental activities. Baidu's annual report on content creation in 2019 showed that the number of short video application users had reached 594 million, accounting for $74.19 \%$ of the total number of Internet users. As of January 2019, the daily live broadcast users of Douyin exceeded 250 million in China, and the monthly live broadcast users exceeded 500 million in China, which continued to grow at a high speed. Faced 
with such a huge user scale, advertisers can't give up this new battlefield. From the perspective of sales scale, media user traffic is the main factor affecting the advertising effect. Such short videos will be a new channel for APP brand communication and product communication on social platforms.

\subsection{The audience's vertical screen usage habits}

According to the "Creative Guidance Manual for Vertical Screen Advertising", the change of video from "horizontal version" to "vertical version” also reflects the change of users' habits. This change is a change in people' s acceptance of video advertisements, from immovable scenes to horizontal videos to vertical videos. Physiologically speaking, human hands can firmly grasp the vertical display design, while horizontal display needs the help of both hands to balance. Vertical screen display allows the user to hold the mobile phone naturally, and the user can shoot and watch in a comfortable posture. Vertical screen short video advertisement can also watch the whole mobile phone screen naturally without deliberately turning the mobile phone, which weakens the user's experience. According to the principle of preference or proportion in communication, the audience chooses the most suitable and labor-saving medium when choosing information. The Douyin of vertical screen short film advertisement is a visible algorithm or hobby based on random push. The audience can get short video advertisement effortlessly. The video switching method is extremely simple and can slide up or down gently, so the audience does not reject the advertisement.

\section{The content and form of vertical screen short video advertising in the era of mobile media}

The dissemination of information is the function and function of advertising. We can regard advertising as a means of communication, and through the function of goods and the promotion of information, consumers can achieve the expected goals. Among many influencing factors, the influence of communication content and form on advertising communication cannot be ignored. Therefore, with the author collecting materials, taking the traditional content and form as the breakthrough point, the study of vertical screen short video advertising communication is as follows.

\subsection{The original biochemistry of vertical screen short video advertising content}

In the development stage of vertical screen short film advertisement, we can see that short video advertisement has main functions, but there is no uniform definition of the main functions of related areas. The author believes that the formation of media and the development of major video advertisements are the foundation. Without media, domestic short video advertisements cannot be produced. At the same time, advertising forms and media environment show the characteristics of unity. Advertising designers will design advertisements according to the platform content, so they are closely related and more private. Excellent content with promotion and viewing value can become a short video platform. At present, the number of short video advertisements with original sound features has even been recognized by users, and has become the function of short video platform content.

Taking Douyin as an example, it cooperates with many enterprises to put vertical short video advertisements on this platform, which will play a role in promotion and become one of the short video social platforms with the most users in China. In order to increase the publicity effect, designers will adopt effective design methods, so as to obtain the unified integration of advertisement and content, and keep the advertisement content consistent with the overall style of the platform. In many cases, users can't even clearly distinguish between advertisement and normal content. Compared with traditional advertisements, this form of advertisement is easier to be accepted and recognized by users, which has important advantages and does not bore users. Advertising is also the calculation result based on big data technology, which can meet the needs of users to a certain extent. The contradiction between users and advertisements will also be solved, and the influence of advertising communication will increase. After watching interesting stories and advertisements, some users also spread them in the circle of friends, thus further expanding the coverage of advertisements. 


\subsection{Vertical screen short video advertisements that have various forms and contents}

At present, vertical short video advertisements on major video social platforms mainly include open-screen advertisements, information flow advertisements, product placement advertisements, shopping cart-type e-commerce marketing advertisements, and live broadcast advertisements. Different advertising styles make viewers feel fresh, give viewers different visual experiences and advertising contents. For example, the form of live broadcast advertisement is vertical screen, which narrows the distance between the audience and the live broadcaster, produces intimacy, and is immersed in the video. Vertical screen viewing can give viewers the illusion of leaving the environment, produce experience in a closed viewing area, and obtain better advertising effect. For example, e-commerce marketing advertisements are generally disliked by the audience, and straightforward products are disgusting. However, vertical short video commercial advertisements have short time, and at the same time, the audience can face the process of product manufacturing or collection, and feel the novel advertising experience. Penguin Intelligence said in "Kuaishou \& Douyin User Research Report” in March, 2018 that users do not reject creative advertisements, but prefer to focus on interesting ordinary people and talented people, and prefer watching video elements, including handsome guys, cute babies, delicious food and pets. Therefore, these forms of short video advertisements with vertical screens in various forms have greatly improved advertising activities.

To sum up, in the era of mobile media, vertical screen short video advertisements have been widely used and achieved good results. Compared with ordinary advertisements, they are in line with users' habits and have local and diversified functions, which is beneficial to users. However, Practically speaking, there is still a gap between Chinese and foreign vertical screen short film advertising forms, which needs professionals to explore and constantly try to promote the reform and development of Chinese marketing methods.

\section{References}

1. Ruan S. The rise of vertical video: The new trend of short video creation in the era of mobile communication (in Chinese). West China Broadcasting TV 2018; (23): 5-6, 12.

2. Wang R, Ma X. "Vertical” screen: An analysis of the new development direction of mobile short video (in Chinese). Media 2019; (11): 54-55. 